\title{
Protective Roles of Adipocytokines and Myokines in Cardiovascular Disease
}

\author{
Noriyuki Ouchi, MD, PhD; Koji Ohashi, MD, PhD; \\ Rei Shibata, MD, PhD; Toyoaki Murohara, MD, PhD
}

\begin{abstract}
Obesity is a major risk factor for progression of cardiovascular disease. Adipose tissue is recognized as an endocrine organ producing various secretory molecules, also known as adipocytokines, and dysregulated production of adipocytokines participates in the pathogenesis of obesity complications, including metabolic dysfunction and cardiovascular disorders. Recent evidence indicates that skeletal muscle also functions as an endocrine organ capable of secreting a number of bioactive substances, also referred to as myokines. Several myokines are involved in metabolic and cardiovascular regulation. This review will discuss the clinical and experimental studies that have investigated the protective role of several adipocytokines and myokines in cardiovascular diseases. (Circ J 2016; 80:
\end{abstract} 2073-2080)

Key Words: Adipocytokines; Adipolin; Cardiovascular diseases; Myokine; Omentin

\section{Adipocytokines}

Cardiovascular disease such as ischemic heart disease is the leading cause of death worldwide., ${ }^{1,2}$ Obesity, in particular excessive accumulation of visceral fat, is one of the major risk factors for various cardiovascular diseases. ${ }^{3-6}$ It is well recognized that fat tissue acts as an endocrine organ that secretes a variety of bioactive molecules, also known as adipocytokines or adipokines. ${ }^{7}$ Most adipocytokines are upregulated by obesity, and these factors are typically pro-inflammatory, thereby leading to the development of obesity complications. The pro-inflammatory adipocytokines include tumor necrosis factor (TNF), interleukin (IL)-6, IL-18 and angiopoietin-like protein $2 .{ }^{7}$ In contrast, there are a few adipocytokines that are downregulated by obese states. These adipocytokines generically display antiinflammatory activity and exert salutary actions on obesity-linked disorders, including cardiovascular disease. ${ }^{7,8}$ The well-known anti-inflammatory adipocytokine is adiponectin, which is specifically expressed in adipose tissue. ${ }^{7}$ The level of circulating adiponectin is reduced in association with metabolic dysfunction and atherosclerotic cardiovascular disease. ${ }^{7}$ Accordingly, an imbalance between pro- and anti-inflammatory adipocytokines under conditions of obesity is involved in the development of metabolic and cardiovascular diseases. Recent research has been performed to identify poorly characterized adipocytokines that protect against metabolic and cardiovascular disorders (Figure 1). These newly identified adipocytokines show a cardiovascular protective function that overlaps with adiponectin. However, the signal transduction mechanisms appear to be different between these adipocytokines.

\section{Adipocytokines With Cardiovascular Protective Properties C1q/TNF-Related Protein (CTRP) 9 The CTRPs are con-} served adiponectin paralogs that contain a collagen-like domain followed by a globular domain similar to compliment factor C1q. ${ }^{9}$ Several CTRPs are abundantly expressed in adipose tissue and serve as adipocytokines that are potentially involved in the regulation of cardiovascular and metabolic functions. CTRP9 exhibits the highest amino acid identity to adiponectin among the CTRPs. ${ }^{9}$ CTRP9 is mainly expressed in adipose tissue, and its circulating levels were decreased in a rodent model of diabetes and obesity.9,10 Several experimental studies have demonstrated that CTRP9 exerts beneficial effects on metabolic and cardiovascular homeostasis. Administration of CTRP9 reduces serum glucose levels in obese mice. ${ }^{9}$ CTRP9 stimulates fatty acid oxidation in skeletal muscle via activation of AMP-activated protein kinase (AMPK). ${ }^{11}$ Transgenic overexpression of CTRP9 results in improvement of high-fat dietinduced obesity, insulin resistance and hepatic steatosis. ${ }^{11}$ Conversely, CTRP9 deficiency results in exacerbation of insulin resistance and hepatic steatosis. ${ }^{12}$ These results indicate the crucial role of CTRP9 in controlling metabolic function. CTRP9 promotes vascular relaxation through the AMPK/endothelial nitric oxide synthase (eNOS)-dependent pathway..$^{13}$ CTRP9 is also reported to reduce inflammatory responses in endothelial cells through the AMPK-dependent pathway. ${ }^{14}$ Moreover, CTRP9 attenuates neointimal proliferative response and formation in mice following vascular injury, and promotes reendothelialization in the injured artery. ${ }^{15}$ The protective effects of CTRP9 on the vascular response to injury are mediated by its ability to suppress vascular smooth muscle cell growth

Received July 7, 2016; revised manuscript received August 3, 2016; accepted August 5, 2016; released online September 1, 2016

Molecular Cardiovascular Medicine (N.O., K.O.), Department of Advanced Cardiovascular Therapeutics (R.S.), and Department of Cardiology (T.M.), Nagoya University Graduate School of Medicine, Nagoya, Japan

Mailing address: Noriyuki Ouchi, MD, PhD, Molecular Cardiovascular Medicine, Nagoya University Graduate School of Medicine, 65

Tsurumai, Showa-ku, Nagoya 466-8550, Japan. E-mail: nouchi@med.nagoya-u.ac.jp

ISSN-1346-9843 doi:10.1253/circj.CJ-16-0663

All rights are reserved to the Japanese Circulation Society. For permissions, please e-mail: cj@j-circ.or.jp 


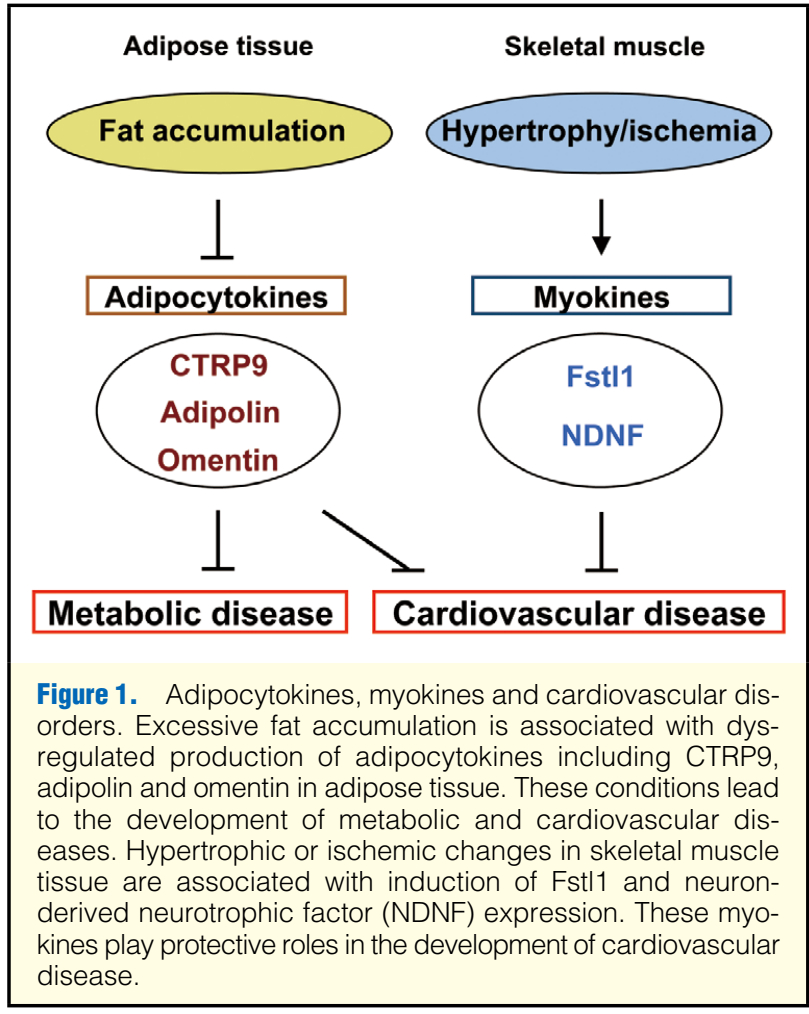

through the cyclic AMP/protein kinase A (PKA)-dependent pathway. In addition, CTRP9 reduces the inflammatory response of macrophages and enhances plaque stability in apolipoprotein E (ApoE)-knockout mice, which are commonly used as models for atherosclerosis. ${ }^{16}$ Thus, CTRP9 may serve as a vasculoprotective adipocytokine.

It has been shown that systemic delivery of CTRP9 reduces myocardial infarct (MI) size, apoptosis and pro-inflammatory gene expression in mice following ischemia-reperfusion. ${ }^{10,17,18}$ Of note, CTRP9 deletion leads to exacerbation of myocardial dysfunction, apoptosis and inflammatory response following ischemia-reperfusion. CTRP9 also ameliorates left ventricular dysfunction in a mouse model of endotoxemia. ${ }^{17}$ In vitro studies showed that CTRP9 attenuates apoptotic activity and inflammatory reaction in cardiac myocytes. ${ }^{10,17}$ The anti-apoptotic and anti-inflammatory effects of CTRP9 in cardiac myocytes are mediated, at least in part, through its ability to promote the AMPK or cyclic AMP signaling pathway. Moreover, CTRP9 improves adverse cardiac remodeling in mice following MI through the PKA-dependent pathway. ${ }^{19}$ Therefore, CTRP9 appears to act as a cardioprotective adipocytokine.

A number of clinical studies indicate the association of circulating CTRP9 with obesity-linked metabolic and vascular disorders. An initial observation showed that low levels of plasma CTRP9 are associated with insulin resistance and abdominal fat accumulation in subjects with normal glucose tolerance and prediabetes/type 2 diabetes. ${ }^{20}$ In contrast, another study demonstrated that elevated CTRP9 concentrations are associated with increased body mass index and insulin resistance in patients with type 2 diabetes, and that CTRP9 levels positively correlate with arterial stiffness. ${ }^{21} \mathrm{~A}$ recent study also demonstrated that serum CTRP9 levels are increased in obese subjects compared with lean subjects, and that weight reduction surgery leads to a decrease in serum CTRP9 level. ${ }^{22}$

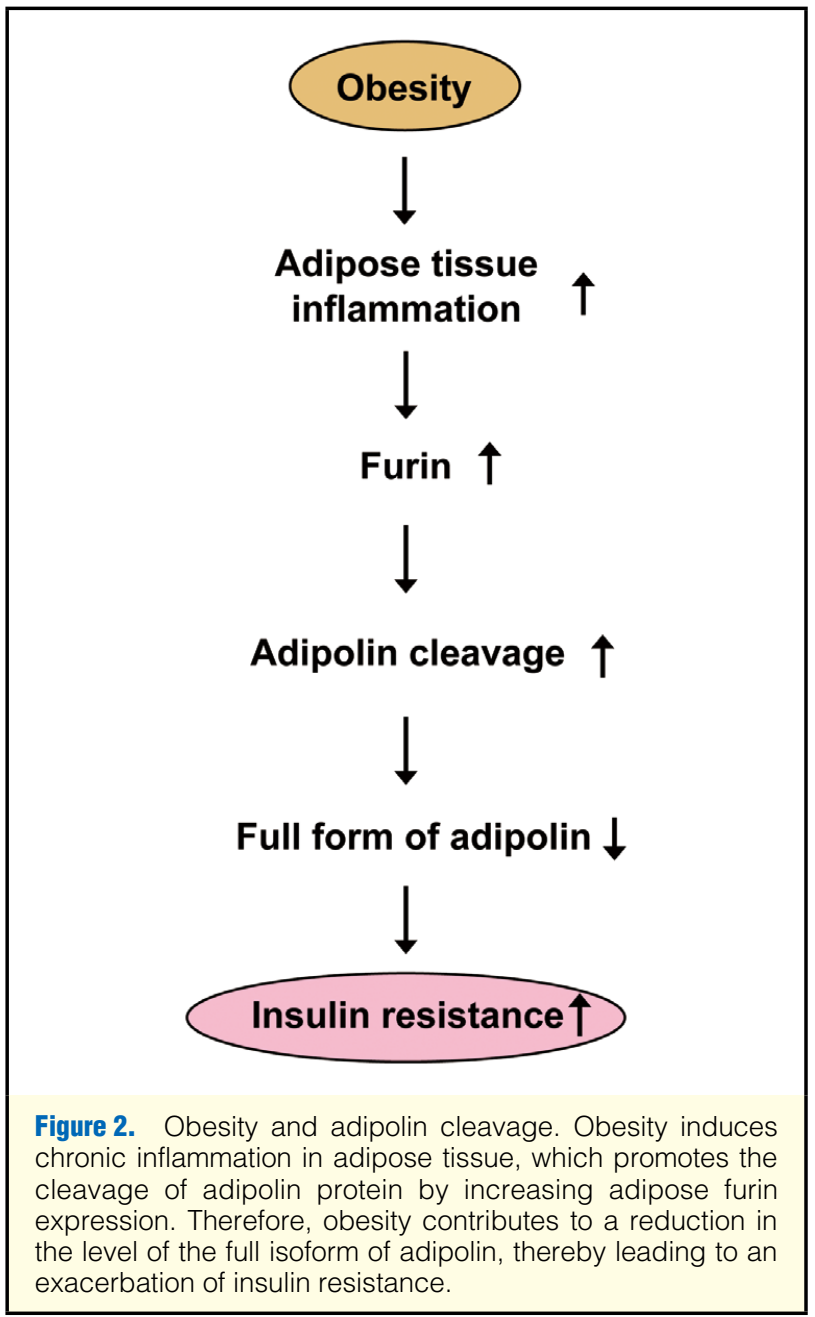

Considering the beneficial effects of CTRP9 on the vasculature and metabolism, increased CTRP9 levels may represent a compensatory response in obese complications. These inconsistent results may be related to the differences in study populations, especially the extent of body mass index and glucose tolerance. Thus, it remains controversial whether CTRP9 is a reliable biomarker for obesity-linked complications, and future studies are needed to elucidate this relationship.

Adipolin/CTRP12 CTRP12 has been identified as a novel adipocytokine with insulin-sensitizing properties and was designated adipolin (adipose-derived insulin-sensitizing factor) to indicate its potential function. ${ }^{23}$ Adipolin is primarily expressed in adipose tissue, in particular, in adipocytes, and its expression is downregulated by obese states. Experimental studies indicate that adipolin acts as a metabolic regulator with antiinflammatory properties. Systemic injection of adipolin ameliorates glucose intolerance and insulin resistance in diet-induced obese mice. ${ }^{23}$ Adipolin treatment also reduces macrophage accumulation and expression of pro-inflammatory genes in fat tissue of obese mice. In cultured macrophages, adipolin attenuates the expression of pro-inflammatory mediators in response to various inflammatory stimuli. Because macrophage inflammation in adipose tissue is associated with the development of insulin resistance, ${ }^{24}$ it is conceivable that adipolin improves insulin sensitivity, at least in part, by reducing macrophage activation in fat tissue. Moreover, adipolin enhances insulin 


\section{Omentin}

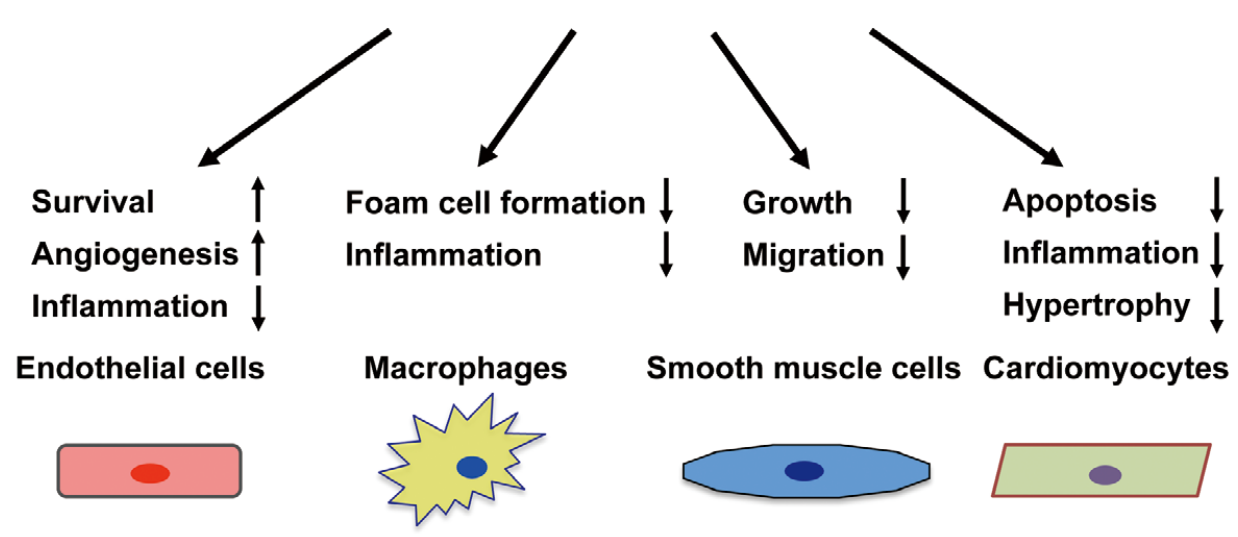

Figure 3. Cardiovascular protection by omentin. Omentin exerts protective actions on the cardiovascular system. It stimulates endothelial cell survival and the angiogenic response, and reduces the inflammatory response of endothelial cells. Omentin reduces foam cell formation by macrophages and suppresses the inflammatory response of macrophages. Omentin also inhibits the growth and migration of vascular smooth muscle cells. Furthermore, omentin reduces apoptosis, inflammation and hypertrophic response in cardiomyocytes.

signaling in the liver and adipose tissue in obese and diabetic mice, thereby leading to amelioration of glucose tolerance and insulin sensitivity. ${ }^{25}$ It is speculated that adipolin plays a role in various obesity-linked inflammatory disorders, including atherosclerosis, and future researches are required to elucidate the involvement of adipolin in the development of cardiovascular disease.

Several studies indicate that adipolin expression is regulated at both transcriptional and post-transcriptional levels. Adipolin expression in adipose tissue and plasma is reduced in both obese ob/ob mice and in diet-induced obese mice. Obesity is associated with chronic low-grade inflammation in adipose tissue. In this regard, adipolin expression is reduced in cultured adipocytes by treatment with the pro-inflammatory cytokine TNF- $\alpha{ }^{23}$ It has been shown that the transcriptional factor Krüppel-like factor (KLF) 15 enhances transcript levels of adipolin in cultured adipocytes. ${ }^{26}$ KLF15 expression is decreased in fat tissue of obese mice and TNF- $\alpha$-treated adipocytes. Thus, it is likely that adipose tissue inflammation caused by obesity leads to decreased expression of KLF15, thereby contributing to reduction of adipose adipolin expression. In contrast to KLF15, KLF3 is shown to negatively regulate adipolin expression. ${ }^{27}$ Thus, approaches for targeting KLF3 and KLF15 may be valuable for the manipulation of adipolin production.

Adipolin is present in blood stream as 2 isoforms (full and cleaved forms). ${ }^{25,28}$ It has been reported that endopeptidase furin cleaves adipolin protein between $91-\mathrm{K}$ and $92-\mathrm{S}$ and generates cleaved form of adipolin. ${ }^{29}$ It has also been shown that diet-induced obese mice exhibit reduction of circulating levels of full and total (full and cleaved) adipolin with an increased ratio of cleaved to full isoforms. ${ }^{28}$ Furin is upregulated in the fat tissue of obese mice and in TNF- $\alpha$-treated adipocytes. Thus, it is conceivable that obesity-induced inflammatory changes in adipose tissue promote the cleavage of adipolin by increasing adipose furin expression (Figure 2).
It has been reported that the full form of adipolin enhances insulin-induced glucose uptake in adipocytes more effectively as compared with its cleaved form. ${ }^{29}$ Those results indicate that obesity contributes to a reduction in circulating levels of the full isoform of adipolin, thereby leading to the development of insulin resistance. Taken together, adipolin, particularly its full isoform, may represent a novel target for prevention or treatment of obesity-linked metabolic and inflammatory diseases.

Omentin Omentin, also known as intelectin-1, is a soluble galactofuranose-binding lectin that has been identified as a circulating adipocytokine. ${ }^{30,31}$ Omentin expression is much higher in human visceral adipose tissue compared with other tissues. ${ }^{31,32}$ A number of epidemiological studies have demonstrated the relationship between omentin and obesity-linked disorders. Decreased levels of circulating omentin are observed in obese subjects, and circulating omentin levels inversely correlate with body mass index, waist circumference and insulin resistance. ${ }^{32}$ Plasma omentin levels are also reduced in patients with impaired glucose tolerance and type 2 diabetes. ${ }^{33}$ A large population-based cohort study showed that circulating omentin levels positively associated with insulin sensitivity. ${ }^{34}$ Furthermore, there is an inverse correlation between omentin levels and the multiplicity of metabolic risk factors, such as increased waist circumference, dyslipidemia, elevated blood pressure, and glucose intolerance, in healthy men. ${ }^{35}$ In contrast, a recent prospective study indicated no significant association of omentin- 1 concentration with the risk of type 2 diabetes. ${ }^{36}$ The reason for these inconsistent results is unclear, and the role of circulating omentin in metabolic dysfunction has yet to be definitely determined.

Moreover, plasma omentin levels are reduced in male patients with coronary artery disease (CAD).${ }^{37}$ Serum omentin levels are negatively associated with the severity of CAD in patients with metabolic syndrome. ${ }^{38}$ Likewise, decreased levels of omentin are associated with CAD and its severity in postmenopausal 
women. ${ }^{39}$ Low levels of circulating omentin are also observed in patients with acute MI. ${ }^{40}$ In addition, omentin levels inversely correlate with carotid intima-media thickness, which is a marker for atherosclerosis. ${ }^{41}$ Furthermore, decreased levels of serum omentin are associated with poor cardiac outcome in patients with heart failure. ${ }^{42}$ Although these findings indicate that omentin may be a useful in the evaluation of cardiovascular disease, further research in different populations is needed to elucidate the clinical significance of this adipocytokine.

Several experimental studies indicate the potential role of omentin in preventing the development of vascular disorders. Omentin enhances endothelial cell survival and network formation via activation of AMPK/eNOS signaling pathways. ${ }^{43}$ Omentin also promotes the endothelial angiogenic response to tissue ischemia in vivo through an eNOS-dependent mechanism. Similarly, omentin induces vascular relaxation through modulation of endothelium-derived NO.44 Omentin is reported to suppress the inflammatory response of cultured endothelial cells, presumably through the AMPK/eNOS-mediated pathways. ${ }^{45}$ Furthermore, omentin inhibits growth factor-stimulated growth and migration of vascular smooth muscle cells. ${ }^{46,47}$ Of note, transgenic mice expressing omentin in fat tissue have decreased neointimal formation and vascular cell proliferation in injured arteries in vivo. ${ }^{46}$ The suppressive effects of omentin on the vascular response to injury are partly dependent on its ability to promote AMPK activation. A recent report showed that overexpression of omentin in fat tissue leads to a reduction of atherosclerotic lesion areas in ApoE-knockout mice, which is accompanied by decreases in macrophage accumulation and pro-inflammatory gene expression. ${ }^{48}$ Consistently, infusion of omentin protein in ApoE-knockout mice reduces the progression of aortic atherosclerotic lesion formation with an accompanying reduction of macrophage content. ${ }^{49}$ Treatment of macrophages with omentin reduces the inflammatory response and transformation into foam cells. Therefore, omentin serves as a vasculoprotective adipocytokine with antiinflammatory properties (Figure 3).

Omentin also plays a protective role in various heart diseases. Systemic administration of omentin to mice results in reduction of MI size and apoptosis following ischemia-reperfusion. ${ }^{50}$ Overexpression of omentin in adipose tissue also attenuates myocardial injury in response to ischemia-reperfusion in mice. The suppressive actions of omentin on myocardial ischemic injury are mediated partly through 2 independent mechanisms involving Akt and AMPK. Furthermore, omentin inhibits cardiac hypertrophy and fibrosis after transverse aortic constriction or angiotensin II infusion. ${ }^{51}$ Omentin reduces the agonist-stimulated hypertrophic response of cardiac myocytes, at least in part, through the AMPK-dependent mechanism. Thus, it is likely that omentin is a cardioprotective adipocytokine (Figure 3). Taken together, decreased levels of circulating omentin caused by obesity may contribute to the development of cardiovascular disorders. However, additional research is needed to dissect the precise signaling cascade of cardiovascular protection induced by omentin.

\section{Myokines}

Skeletal muscle is one of the largest of the organs that regulate energy and metabolic homeostasis. It is commonly known that exercise training leads to improvements in whole-body metabolism. Conventional endurance exercise training benefits the reduction of risk factors for cardiovascular disease and cardiovascular mortality and morbidity. ${ }^{52-54}$ It has also been shown that resistance exercise training, which is associated with mus- cle hypertrophy and strengthening, exerts beneficial actions on the cardiovascular system. ${ }^{55,56}$ Recent evidence indicates that skeletal muscle acts as an endocrine organ capable of secreting various bioactive molecules, also referred to as myokines, which can directly affect nearby or remote tissues. ${ }^{57}$ It has been proposed that the well-known protective actions of exercise are partly mediated by increased secretion of myokines. ${ }^{57}$ In this regard, myokines are considered to represent novel targets for treatments that mimic the benefits of exercise training for metabolic dysfunction and cardiovascular disorders. Therefore, the notion that myokines function as modulators of whole-body homeostasis has raised much attention in the field of medical research.

IL-6 has been identified as a myokine that is dramatically induced during physical exercise. ${ }^{57}$ The beneficial effects of exercise on glucose metabolism and inflammation are partly mediated by muscle-derived IL- $6 .{ }^{57} \mathrm{IL}-15$ was identified as a myokine that is induced by resistance training. $.^{58} \mathrm{IL}-15$ derived from skeletal muscle negatively regulates visceral fat mass. ${ }^{59}$ Myostatin was identified as a muscle-specific secretory factor that regulates skeletal muscle mass in an autocrine manner. ${ }^{60}$ Myostatin was shown to also reduce fat tissue mass in an endocrine manner. ${ }^{61}$ Irisin is a PPAR- $\gamma$ co-activator- $1-\alpha$-dependent myokine that drives brown-fat-like development of white fat, and is involved in the regulation of obesity and glucose homeostasis. ${ }^{62}$ These myokines appear to participate in maintenance of whole-body metabolic homeostasis.

Sarcopenia is an age-related condition that is characterized by skeletal mass loss and dysfunction. Some myokines have been proposed as potential biomarkers of sarcopenia. Serum myostatin concentrations positively correlate with muscle mass, and increased myostatin levels are observed in elderly patients with muscle wasting. ${ }^{63}$ In contrast, serum concentrations of myostatin do not differ between young men and sarcopenic elderly men. ${ }^{64}$ Similarly, serum levels of myostatin do not reflect the presence of sarcopenia in elderly women. ${ }^{65}$ It has been shown that serum irisin level is associated with sarcopenia in peritoneal dialysis patients. ${ }^{66}$ By contrast, circulating irisin levels are not different in individuals with sarcopenia compared with control subjects. ${ }^{67}$ Thus, the relationship between circulating levels of these myokines and sarcopenia appears to be limited and contradictory, and this requires further investigation.

Recent research has been performed to discover novel myokines involved in cardiovascular regulation (Figure 1). Follistatin-like 1 (Fstl1) has been identified as a myokine that is upregulated in hypertrophic muscle in mice. ${ }^{68}$ Fstl1 protects against vascular injury, cardiac injury and myocardial hypertrophic response. ${ }^{68-70}$ Neuron-derived neurotrophic factor (NDNF)/epidermacan was identified as a secreted molecule that is upregulated in ischemic limbs of mice. ${ }^{71} \mathrm{NDNF}$ is reported to prevent endothelial cell injury and the adverse cardiac remodeling in response to ischemia. ${ }^{72}$ Although most of the previously studied myokines act as metabolic regulators, these myokines appear to exert cardiovascular protective effects as described next in detail.

\section{Myokines With Cardiovascular Protective Properties}

Fstl1 Fstl1, also referred to as TSC-36, is a secreted glycoprotein that belongs to the follistatin family of proteins. Fstl1 was identified as one of secretory factors that are upregulated during skeletal muscle hypertrophy in transgenic mice expressing Akt1 in a skeletal muscle-specific manner ${ }^{68}$ (Figure 4). Fstl1 expression is also increased in ischemic skeletal muscle of mice ${ }^{68}$ (Figure 4). Fstl1 promotes differen- 
tiation of endothelial cells into network structures and inhibits apoptosis. ${ }^{68}$ Intramuscular injection of Fstl1 also stimulates revascularization processes in response to ischemia in mice. The vascular effects of Fstl1 are mediated partly through its ability to modulate eNOS activation. ${ }^{68}$ Moreover, targeted ablation of Fstl1 in muscle results in an increase in neointimal formation following wire-induced arterial injury. ${ }^{73}$ Conversely, muscle-specific overexpression of Fstl1 decreases neointimal thickening in response to injury. Fstl1 reduces the proliferation of vascular smooth muscle cells partly via activation of AMPK. Thus, it is likely that Fstl1 functions as a myokine that can modulate endothelial cell apoptosis, vascular smooth muscle cell proliferation and neointimal formation (Figure 4).

Fstl1 is abundantly expressed in the heart as well as in skeletal muscle and is secreted by cardiomyocytes. ${ }^{69}$ Fstl1 expression is increased in ischemia-injured and hypertrophic hearts of mice. ${ }^{69}$ Systemic administration of Fstl1 to mice leads to a reduction of MI size following ischemia-reperfusion, which is accompanied by reduced apoptosis and inflammatory response in the ischemic heart. ${ }^{69,74}$ Furthermore, intracoronary administration of Fstl1 protein improves cardiac damage and function in a pig model of ischemia-reperfusion. ${ }^{74}$ Thus, it is conceivable that Fstl1 could be a novel therapeutic target for acute ischemic injury in the heart. Treatment of cultured cardiac myocytes with Fstl1 reduces apoptosis in response to hypoxia-reoxygenation through its ability to activate Akt and AMPK, and antagonize bone morphogenetic protein (BMP)-4 signaling. ${ }^{69,74}$ Fstl1 also suppresses inflammatory response of cardiac myocytes, at least in part, through its ability to promote AMPK activation and inhibit BMP-4 pathway. ${ }^{74} \mathrm{~A}$ recent study showed that epicardial Fstl1 reconstitution stimulates cell cycle entry and division of pre-existing cardiomyocytes, resulting in improvement of cardiac function and survival in mouse and swine models of MI. ${ }^{75}$ A more recent study demonstrated that Fstl1 stimulates early fibroblast activation, which is required for acute repair of the infarcted heart, and protects the heart from rupture in a mouse model of MI. ${ }^{76}$ These observations suggest that Fstl1 modulates the myocardial response to ischemic insult, thereby leading to cardioprotection (Figure 4). It has also been reported that cardiac-specific Fstl1-knockout mice have enhanced cardiac hypertrophy following pressure overload compared with control mice, ${ }^{70}$ indicating that Fstl1 derived from cardiomyocytes can function to reduce the myocardial hypertrophic response. A recent report showed that Fstl1 protects against kidney injury in a model of chronic renal failure. ${ }^{77}$ Cardiac-specific Fstl1-knockout mice show a significant reduction in circulating Fstll levels after subtotal nephrectomy compared with control mice, suggesting that cardiomyocytes are one of the main sources of circulating Fstl1 during renal injury. Of note, ablation of Fstl1 in a cardiac myocyte-specific manner leads to exacerbation of urinary albumin excretion, glomerular hypertrophy, renal inflammatory response and tubulointerstitial fibrosis after subtotal nephrectomy, indicating that cardiomyocyte-derived Fstl1 plays a crucial role in preventing chronic kidney disease. Taken together, Fstl1 functions as a cardiovascular and renal protective factor secreted by skeletal muscle and heart via an autocrine, paracrine or endocrine mechanism.

Several epidemiological studies have demonstrated the association between Fstl1 and cardiovascular disease. Circulating Fstl1 levels are increased in patients with acute coronary syndrome. ${ }^{78}$ Increased serum Fstl1 levels are associated with left ventricular hypertrophy in patients with systolic heart failure, and Fstl1 expression is enhanced in the human failing heart. ${ }^{79}$ Plasma Fstll levels are positively associated with inflam-

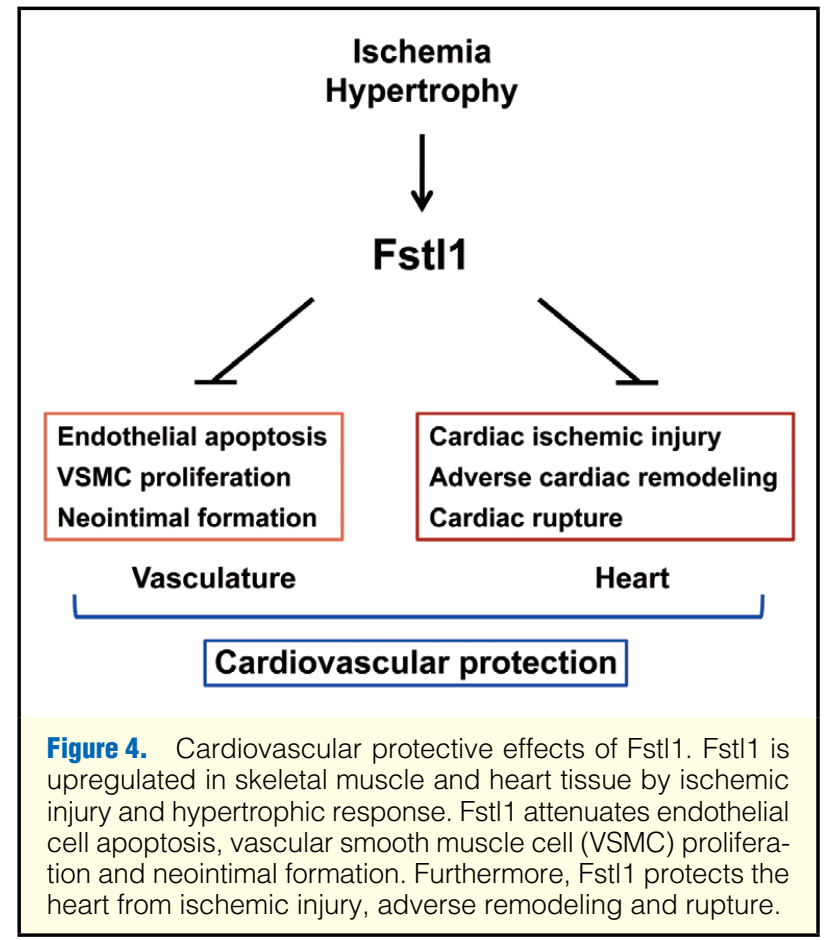

matory and oxidative stress markers in healthy subjects. ${ }^{80}$ Fstl1 secretion from human primary skeletal muscle cells is enhanced by treatment with interferon- $\gamma$ and IL- $1 \beta,{ }^{81}$ suggesting that inflammatory and oxidative stress conditions lead to induction of Fstl1 expression. Considering the experimental data, increased levels of circulating Fstl1 may a compensatory response to mitigating damage to the cardiovascular system. Future research on the role of Fstl1 as a useful biomarker for various cardiovascular diseases is warranted.

NDNF NDNF is a glycosylated secreted protein that has a fibronectin type III domain. ${ }^{82}$ NDNF was initially identified as a neurotrophic factor expressed in mouse brain and spinal cord, and it promotes migration and neurite growth of mouse hippocampal neurons. ${ }^{82}$ Recent research has been aimed at identifying poorly characterized myokines that are involved in ischemia-induced vessel growth. NDNF was selected as one of potential secreted molecules that are upregulated by ischemia after screening of differentially regulated genes in nonischemic and ischemic muscle tissues of mice. ${ }^{71}$ Interestingly, NDNF expression is observed in endothelial cells rather than in myocytes in skeletal muscle tissue. ${ }^{71}$ Ischemic insult in the hindlimb leads to enhanced expression in muscle, which is accompanied by increased levels of circulating NDNF. NDNF secretion from endothelial cells is enhanced in response to hypoxia. NDNF stimulates endothelial cell network formation and survival through activation of Akt/eNOS signaling pathway. Conversely, knockdown of NDNF in endothelial cells results in diminished network formation and enhanced apoptotic activity. These data suggest that NDNF is a hypoxiainducible pro-angiogenic factor secreted by endothelial cells. Intramuscular injection of NDNF enhances blood flow recovery and capillary formation in ischemic limbs of mice. ${ }^{71}$ Moreover, downregulation of muscle NDNF by siRNA technique leads to reduction of perfusion recovery in response to ischemia. Thus, NDNF serves as an endogenous ischemia-inducible factor secreted from skeletal muscle that can enhance endothe- 
lial cell function and ischemia-induced revascularization.

A recent report demonstrated the role of muscle-derived NDNF in regulation of cardiac remodeling. Intramuscular administration of NDNF using an adenoviral vector leads to elevation of circulating NDNF and improvement of cardiac systolic dysfunction in a mouse model of MI. ${ }^{72}$ The salutary actions of NDNF on cardiac function are associated with enhanced capillary formation and reduced myocardial hypertrophy and apoptosis in post-MI hearts. In cultured cardiac myocytes, NDNF reduces hypoxia-induced apoptosis via activation of focal adhesion kinase/Akt-dependent pathway. ${ }^{72}$ NDNF expression is much higher in skeletal muscle than in heart in mice. Thus, NDNF can ameliorate adverse cardiac remodeling after ischemia through its abilities to modulate myocardial apoptosis and angiogenesis by directly influencing cardiomyocyte and endothelial cell function in the heart in an endocrine manner, but additional studies are needed to clarify the role of endogenous NDNF in ischemic cardiovascular disorders using genetically manipulated mice.

\section{Conclusions}

Several adipocytokines and myokines with cardiovascular protective actions have been identified during the past decade. These secretory factors potentially play crucial roles in intertissue communication, and changes in their production in blood stream may participate in cardiovascular homeostasis and disease. In this context, dysregulation of adipocytokines, including CTRP9, adipolin and omentin, under conditions of obesity may contribute to the pathogenesis of cardiovascular and metabolic disorders (Figure 1). Thus, the approaches to modulate the synthesis and secretion of these adipocytokines and myokines or to enhance their signaling cascades could be useful for prevention or treatment of cardiovascular disorders. Furthermore, future elucidation of the functions, molecular mechanisms and regulations of these factors will be needed for the better understanding of pathogenesis of cardiovascular disease.

\section{Acknowledgments}

This work was supported by Grant-in-Aid for Scientific Research (\#26293185) and grants from Takeda Science Foundation and the Uehara Memorial Foundation to N.O.

\section{Conflict of Interest}

Research grants were received from Astellas Pharma Inc. and Daiichi Sankyo Co Ltd (to N.O.). However, the research topics of these grants were not restricted. Lecture fees were received from Kowa Pharmaceutical Co Ltd (to N.O.). Molecular Cardiovascular Medicine was endowed by Kowa Pharmaceutical Co Ltd (to N.O. and K.O.).

\section{References}

1. Roger VL, Go AS, Lloyd-Jones DM, Adams RJ, Berry JD, Brown $\mathrm{TM}$, et al. Heart disease and stroke statistics - 2011 update: A report from the American Heart Association. Circulation 2011; 123: e18e209, doi:10.1161/CIR.0b013e3182009701.

2. Ohira T, Iso H. Cardiovascular disease epidemiology in Asia: An overview. Circ J 2013; 77: 1646-1652.

3. Matsuzawa Y, Funahashi T, Kihara S, Shimomura I. Adiponectin and metabolic syndrome. Arterioscler Thromb Vasc Biol 2004; 24: $29-33$.

4. Nguyen HN, Fujiyoshi A, Abbott RD, Miura K. Epidemiology of cardiovascular risk factors in Asian countries. Circ $J$ 2013; 77: 2851-2859.

5. Yatsuya H, Li Y, Hilawe EH, Ota A, Wang C, Chiang C, et al. Global trend in overweight and obesity and its association with cardiovascular disease incidence. Circ J 2014; 78: 2807-2818.

6. Tsushima H, Yamamoto H, Kitagawa T, Urabe Y, Tatsugami F,
Awai K, et al. Association of epicardial and abdominal visceral adipose tissue with coronary atherosclerosis in patients with a coronary artery calcium score of zero. Circ J 2015; 79: 1084-1091.

7. Ouchi N, Parker JL, Lugus JJ, Walsh K. Adipokines in inflammation and metabolic disease. Nat Rev Immunol 2011; 11: 85-97.

8. Kamata S, Miyagawa S, Fukushima S, Imanishi Y, Saito A, Maeda $\mathrm{N}$, et al. Targeted delivery of adipocytokines into the heart by induced adipocyte cell-sheet transplantation yields immune tolerance and functional recovery in autoimmune-associated myocarditis in rats. Circ J 2015; 79: 169-179.

9. Wong GW, Krawczyk SA, Kitidis-Mitrokostas C, Ge G, Spooner E, Hug C, et al. Identification and characterization of CTRP9, a novel secreted glycoprotein, from adipose tissue that reduces serum glucose in mice and forms heterotrimers with adiponectin. FASEB J 2009; 23: $241-258$.

10. Kambara T, Ohashi K, Shibata R, Ogura Y, Maruyama S, Enomoto $\mathrm{T}$, et al. CTRP9 protein protects against myocardial injury following ischemia-reperfusion through AMP-activated protein kinase (AMPK)dependent mechanism. J Biol Chem 2012; 287: 18965-18973.

11. Peterson JM, Wei Z, Seldin MM, Byerly MS, Aja S, Wong GW. CTRP9 transgenic mice are protected from diet-induced obesity and metabolic dysfunction. Am J Physiol Regul Integr Comp Physiol 2013; 305: R522-R533.

12. Wei Z, Lei X, Petersen PS, Aja S, Wong GW. Targeted deletion of $\mathrm{C} 1 \mathrm{q} / \mathrm{TNF}$-related protein 9 increases food intake, decreases insulin sensitivity, and promotes hepatic steatosis in mice. Am J Physiol Endocrinol Metab 2014; 306: E779-E790, doi:10.1152/ajpendo. 00593.2013.

13. Zheng Q, Yuan Y, Yi W, Lau WB, Wang Y, Wang X, et al. C1q/ TNF-related proteins, a family of novel adipokines, induce vascular relaxation through the adiponectin receptor-1/AMPK/eNOS/nitric oxide signaling pathway. Arterioscler Thromb Vasc Biol 2011; 31: $2616-2623$.

14. Jung CH, Lee MJ, Kang YM, La Lee Y, Seol SM, Yoon HK, et al. C1q/TNF-related protein-9 inhibits cytokine-induced vascular inflammation and leukocyte adhesiveness via AMP-activated protein kinase activation in endothelial cells. Mol Cell Endocrinol 2016; 419: $235-243$.

15. Uemura Y, Shibata R, Ohashi K, Enomoto T, Kambara T, Yamamoto T, et al. Adipose-derived factor CTRP9 attenuates vascular smooth muscle cell proliferation and neointimal formation. FASEB J 2013; 27: $25-33$.

16. Li J, Zhang P, Li T, Liu Y, Zhu Q, Chen T, et al. CTRP9 enhances carotid plaque stability by reducing pro-inflammatory cytokines in macrophages. Biochem Biophys Res Commun 2015; 458: 890-895.

17. Kambara T, Shibata R, Ohashi K, Matsuo K, Hiramatsu-Ito M, Enomoto T, et al. C1q/tumor necrosis factor-related protein 9 protects against acute myocardial injury through an adiponectin receptor I-AMPK-dependent mechanism. Mol Cell Biol 2015; 35: 2173 2185.

18. Su H, Yuan Y, Wang XM, Lau WB, Wang Y, Wang X, et al. Inhibition of CTRP9, a novel and cardiac-abundantly expressed cell survival molecule, by TNFalpha-initiated oxidative signaling contributes to exacerbated cardiac injury in diabetic mice. Basic Res Cardiol 2013; 108: 315 .

19. Sun Y, Yi W, Yuan Y, Lau WB, Yi D, Wang X, et al. C1q/tumor necrosis factor-related protein-9, a novel adipocyte-derived cytokine, attenuates adverse remodeling in the ischemic mouse heart via protein kinase A activation. Circulation 2013; 128: S113-S120.

20. Hwang YC, Woo Oh S, Park SW, Park CY. Association of serum C1q/TNF-related protein-9 (CTRP9) concentration with visceral adiposity and metabolic syndrome in humans. Int $J$ Obes (Lond) 2014; 38: $1207-1212$.

21. Jung CH, Lee MJ, Kang YM, Jang JE, Leem J, Lee YL, et al. Association of serum $\mathrm{C} 1 \mathrm{q} / \mathrm{TNF}$-related protein-9 concentration with arterial stiffness in subjects with type 2 diabetes. J Clin Endocrinol Metab 2014; 99: E2477-E2484, doi:10.1210/jc.2014-2524.

22. Wolf RM, Steele KE, Peterson LA, Zeng X, Jaffe AE, Schweitzer MA, et al. C1q/TNF-related protein-9 (CTRP9). J Clin Endocrinol Metab 2016; 101: 2211-2217.

23. Enomoto T, Ohashi K, Shibata R, Higuchi A, Maruyama S, Izumiya $\mathrm{Y}$, et al. Adipolin/C1qde2/CTRP12 protein functions as an adipokine that improves glucose metabolism. J Biol Chem 2011; 286: 34552 34558 .

24. Sun K, Kusminski CM, Scherer PE. Adipose tissue remodeling and obesity. J Clin Invest 2011; 121: 2094-2101.

25. Wei Z, Peterson JM, Lei X, Cebotaru L, Wolfgang MJ, Baldeviano GC, et al. C1q/TNF-related protein-12 (CTRP12), a novel adipokine that improves insulin sensitivity and glycemic control in mouse models of obesity and diabetes. J Biol Chem 2012; 287: 10301 - 
10315.

26. Enomoto T, Ohashi K, Shibata R, Kambara T, Uemura Y, Yuasa D, et al. Transcriptional regulation of an insulin-sensitizing adipokine adipolin/CTRP12 in adipocytes by Kruppel-like factor 15. PLoS One 2013; 8: e83183, doi:10.1371/journal.pone.0083183.

27. Bell-Anderson KS, Funnell AP, Williams H, Mat Jusoh H, Scully T, Lim WF, et al. Loss of Kruppel-like factor 3 (KLF3/BKLF) leads to upregulation of the insulin-sensitizing factor adipolin (FAM132A/ CTRP12/C1qdc2). Diabetes 2013; 62: 2728-2737.

28. Enomoto T, Shibata R, Ohashi K, Kambara T, Kataoka Y, Uemura Y, et al. Regulation of adipolin/CTRP12 cleavage by obesity. Biochem Biophys Res Commun 2012; 428: 155-159.

29. Wei Z, Lei X, Seldin MM, Wong GW. Endopeptidase cleavage generates a functionally distinct isoform of C1q/tumor necrosis factor-related protein-12 (CTRP12) with an altered oligomeric state and signaling specificity. J Biol Chem 2012; 287: 35804-35814.

30. Tsuji S, Uehori J, Matsumoto M, Suzuki Y, Matsuhisa A, Toyoshima $\mathrm{K}$, et al. Human intelectin is a novel soluble lectin that recognizes galactofuranose in carbohydrate chains of bacterial cell wall. J Biol Chem 2001; 276: 23456-23463.

31. Yang RZ, Lee MJ, Hu H, Pray J, Wu HB, Hansen BC, et al. Identification of omentin as a novel depot-specific adipokine in human adipose tissue: Possible role in modulating insulin action. Am J Physiol Endocrinol Metab 2006; 290: E1253-E1261, doi:10.1152/ ajpendo.00572.2004.

32. de Souza Batista CM, Yang RZ, Lee MJ, Glynn NM, Yu DZ, Pray $\mathrm{J}$, et al. Omentin plasma levels and gene expression are decreased in obesity. Diabetes 2007; 56: 1655-1661.

33. Pan HY, Guo L, Li Q. Changes of serum omentin-1 levels in normal subjects and in patients with impaired glucose regulation and with newly diagnosed and untreated type 2 diabetes. Diabetes Res Clin Pract 2010; 88: $29-33$.

34. Herder C, Ouwens DM, Carstensen M, Kowall B, Huth C, Meisinger $\mathrm{C}$, et al. Adiponectin may mediate the association between omentin, circulating lipids and insulin sensitivity: Results from the KORA F4 study. Eur J Endocrinol 2015; 172: 423-432.

35. Shibata R, Ouchi N, Takahashi R, Terakura Y, Ohashi K, Ikeda N, et al. Omentin as a novel biomarker of metabolic risk factors. Diabetol Metab Syndr 2012; 4: 37.

36. Wittenbecher C, Menzel J, Carstensen-Kirberg M, Biemann R, di Giuseppe R, Fritsche A, et al. Omentin-1, adiponectin, and the risk of developing type 2 diabetes. Diabetes Care 2016; 39: e79-e80, doi:10.2337/dc15-2702.

37. Shibata R, Ouchi N, Kikuchi R, Takahashi R, Takeshita K, Kataoka $\mathrm{Y}$, et al. Circulating omentin is associated with coronary artery disease in men. Atherosclerosis 2011; 219: 811-814.

38. Shang FJ, Wang JP, Liu XT, Zheng QS, Xue YS, Wang B, et al. Serum omentin-1 levels are inversely associated with the presence and severity of coronary artery disease in patients with metabolic syndrome. Biomarkers 2011; 16: 657-662.

39. Onur I, Oz F, Yildiz S, Oflaz H, Sigirci S, Elitok A, et al. Serum omentin 1 level is associated with coronary artery disease and its severity in postmenopausal women. Angiology 2014; 65: 896-900.

40. Kadoglou NP, Tahmatzidis DK, Giannakoulas C, Kapelouzou A, Gkontopoulos A, Parissis J, et al. Serum levels of novel adipokines, omentin-1 and chemerin, in patients with acute myocardial infarction: KOZANI study. J Cardiovasc Med (Hagerstown) 2015; 16: $341-346$.

41. Shibata R, Takahashi R, Kataoka Y, Ohashi K, Ikeda N, Kihara S, et al. Association of a fat-derived plasma protein omentin with carotid artery intima-media thickness in apparently healthy men. Hypertens Res 2011; 34: 1309-1312.

42. Narumi T, Watanabe T, Kadowaki S, Kinoshita D, Yokoyama M, Honda Y, et al. Impact of serum omentin-1 levels on cardiac prognosis in patients with heart failure. Cardiovasc Diabetol 2014; 13: 84.

43. Maruyama S, Shibata R, Kikuchi R, Izumiya Y, Rokutanda T, Araki $\mathrm{S}$, et al. Fat-derived factor omentin stimulates endothelial cell function and ischemia-induced revascularization via endothelial nitric oxide synthase-dependent mechanism. J Biol Chem 2012; 287: $408-417$

44. Yamawaki H, Tsubaki N, Mukohda M, Okada M, Hara Y. Omentin, a novel adipokine, induces vasodilation in rat isolated blood vessels. Biochem Biophys Res Commun 2010; 393: 668-672.

45. Yamawaki H, Kuramoto J, Kameshima S, Usui T, Okada M, Hara Y. Omentin, a novel adipocytokine inhibits TNF-induced vascular inflammation in human endothelial cells. Biochem Biophys Res Commun 2011; 408: 339-343.

46. Uemura Y, Shibata R, Kanemura N, Ohashi K, Kambara T, Hiramatsu-Ito M, et al. Adipose-derived protein omentin prevents neointimal formation after arterial injury. FASEB $J$ 2015; 29: 141 151.

47. Kazama K, Okada M, Yamawaki H. A novel adipocytokine, omentin, inhibits platelet-derived growth factor-BB-induced vascular smooth muscle cell migration through antioxidative mechanism. Am J Physiol Heart Circ Physiol 2014; 306: H1714-H1719.

48. Hiramatsu-Ito M, Shibata R, Ohashi K, Uemura Y, Kanemura N, Kambara $\mathrm{T}$, et al. Omentin attenuates atherosclerotic lesion formation in apolipoprotein E-deficient mice. Cardiovasc Res 2016; 110: $107-117$.

49. Watanabe K, Watanabe R, Konii H, Shirai R, Sato K, Matsuyama TA, et al. Counteractive effects of omentin-1 against atherogenesis ${ }^{\dagger}$. Cardiovasc Res 2016; 110: 118-128.

50. Kataoka Y, Shibata R, Ohashi K, Kambara T, Enomoto T, Uemura $\mathrm{Y}$, et al. Omentin prevents myocardial ischemic injury through AMP-activated protein kinase- and Akt-dependent mechanisms. $J$ Am Coll Cardiol 2014; 63: 2722-2733.

51. Matsuo K, Shibata R, Ohashi K, Kambara T, Uemura Y, HiramatsuIto $\mathrm{M}$, et al. Omentin functions to attenuate cardiac hypertrophic response. J Mol Cell Cardiol 2015; 79: 195-202.

52. Umpierre D, Ribeiro PA, Kramer CK, Leitao CB, Zucatti AT, Azevedo MJ, et al. Physical activity advice only or structured exercise training and association with $\mathrm{HbA} 1 \mathrm{c}$ levels in type 2 diabetes: A systematic review and meta-analysis. JAMA 2011; 305: 17901799 .

53. Morita N, Okita K. Is gender a factor in the reduction of cardiovascular risks with exercise training? Circ J 2013; 77: 646-651

54. Miura Y, Fukumoto Y, Miura T, Shimada K, Asakura M, Kadokami $\mathrm{T}$, et al. Impact of physical activity on cardiovascular events in patients with chronic heart failure: A multicenter prospective cohort study. Circ J 2013; 77: 2963-2972.

55. Heffernan KS, Fahs CA, Iwamoto GA, Jae SY, Wilund KR, Woods $\mathrm{JA}$, et al. Resistance exercise training reduces central blood pressure and improves microvascular function in African American and white men. Atherosclerosis 2009; 207: 220-226.

56. Anton MM, Cortez-Cooper MY, DeVan AE, Neidre DB, Cook JN, Tanaka H. Resistance training increases basal limb blood flow and vascular conductance in aging humans. J Appl Physiol (1985) 2006; 101: $1351-1355$.

57. Pedersen BK, Fischer CP. Beneficial health effects of exercise--the role of IL-6 as a myokine. Trends Pharmacol Sci 2007; 28: $152-$ 156.

58. Nielsen AR, Mounier R, Plomgaard P, Mortensen OH, Penkowa M, Speerschneider T, et al. Expression of interleukin-15 in human skeletal muscle effect of exercise and muscle fibre type composition. $J$ Physiol 2007; 584: 305-312.

59. Nielsen AR, Hojman P, Erikstrup C, Fischer CP, Plomgaard P, Mounier R, et al. Association between interleukin-15 and obesity: Interleukin-15 as a potential regulator of fat mass. J Clin Endocrinol Metab 2008; 93: 4486-4493.

60. McPherron AC, Lawler AM, Lee SJ. Regulation of skeletal muscle mass in mice by a new TGF-b superfamily member. Nature 1997 ; 387: $83-90$.

61. McPherron AC, Lee SJ. Suppression of body fat accumulation in myostatin-deficient mice. J Clin Invest 2002; 109: 595-601.

62. Bostrom P, Wu J, Jedrychowski MP, Korde A, Ye L, Lo JC, et al. A PGC1-alpha-dependent myokine that drives brown-fat-like development of white fat and thermogenesis. Nature 2012; 481: 463-468.

63. Yarasheski KE, Bhasin S, Sinha-Hikim I, Pak-Loduca J, GonzalezCadavid NF. Serum myostatin-immunoreactive protein is increased in 60-92 year old women and men with muscle wasting. $J$ Nutr Health Aging 2002; 6: 343-348.

64. Ratkevicius A, Joyson A, Selmer I, Dhanani T, Grierson C, Tommasi $\mathrm{AM}$, et al. Serum concentrations of myostatin and myostatin-interacting proteins do not differ between young and sarcopenic elderly men. J Gerontol A Biol Sci Med Sci 2011; 66: 620-626.

65. Hofmann M, Halper B, Oesen S, Franzke B, Stuparits P, Tschan H, et al. Serum concentrations of insulin-like growth factor-1, members of the TGF-beta superfamily and follistatin do not reflect different stages of dynapenia and sarcopenia in elderly women. Exp Gerontol 2015; 64: 35-45.

66. Lee MJ, Lee SA, Nam BY, Park S, Lee SH, Ryu HJ, et al. Irisin, a novel myokine is an independent predictor for sarcopenia and carotid atherosclerosis in dialysis patients. Atherosclerosis 2015; 242: 476482.

67. Choi HY, Kim S, Park JW, Lee NS, Hwang SY, Huh JY, et al. Implication of circulating irisin levels with brown adipose tissue and sarcopenia in humans. J Clin Endocrinol Metab 2014; 99: $2778-$ 2785.

68. Ouchi N, Oshima Y, Ohashi K, Higuchi A, Ikegami C, Izumiya Y, 
et al. Follistatin-like 1, a secreted muscle protein, promotes endothelial cell function and revascularization in ischemic tissue through a nitric-oxide synthase-dependent mechanism. J Biol Chem 2008; 283: 32802-32811.

69. Oshima Y, Ouchi N, Sato K, Izumiya Y, Pimentel DR, Walsh K. Follistatin-like 1 is an Akt-regulated cardioprotective factor that is secreted by the heart. Circulation 2008; 117: 3099-3108.

70. Shimano M, Ouchi N, Nakamura K, van Wijk B, Ohashi K, Asaumi $\mathrm{Y}$, et al. Cardiac myocyte follistatin-like 1 functions to attenuate hypertrophy following pressure overload. Proc Natl Acad Sci USA 2011; 108: E899-E906, doi:10.1073/pnas.1108559108.

71. Ohashi K, Enomoto T, Joki Y, Shibata R, Ogura Y, Kataoka Y, et al. Neuron-derived neurotrophic factor functions as a novel modulator that enhances endothelial cell function and revascularization processes. J Biol Chem 2014; 289: 14132-14144.

72. Joki Y, Ohashi K, Yuasa D, Shibata R, Kataoka Y, Kambara T, et al. Neuron-derived neurotrophic factor ameliorates adverse cardiac remodeling after experimental myocardial infarction. Circ Heart Fail 2015; 8: $342-351$.

73. Miyabe M, Ohashi K, Shibata R, Uemura Y, Ogura Y, Yuasa D, et al. Muscle-derived follistatin-like 1 functions to reduce neointimal formation after vascular injury. Cardiovasc Res 2014; 103: 111120.

74. Ogura Y, Ouchi N, Ohashi K, Shibata R, Kataoka Y, Kambara T, et al. Therapeutic impact of follistatin-like 1 on myocardial ischemic injury in preclinical models. Circulation 2012; 126: 1728-1738.

75. Wei K, Serpooshan V, Hurtado C, Diez-Cunado M, Zhao M, Maruyama S, et al. Epicardial FSTL1 reconstitution regenerates the adult mammalian heart. Nature 2015; 525: 479-485.
76. Maruyama S, Nakamura K, Papanicolaou KN, Sano S, Shimizu I, Asaumi Y, et al. Follistatin-like 1 promotes cardiac fibroblast activation and protects the heart from rupture. EMBO Mol Med 2016; 8: 949-966.

77. Hayakawa S, Ohashi K, Shibata R, Kataoka Y, Miyabe M, Enomoto $\mathrm{T}$, et al. Cardiac myocyte-derived follistatin-like 1 prevents renal injury in a subtotal nephrectomy model. J Am Soc Nephrol 2015; 26: 636-646.

78. Widera C, Horn-Wichmann R, Kempf T, Bethmann K, Fiedler B, Sharma S, et al. Circulating concentrations of follistatin-like 1 in healthy individuals and patients with acute coronary syndrome as assessed by an immunoluminometric sandwich assay. Clin Chem 2009; 55: 1794-1800.

79. El-Armouche A, Ouchi N, Tanaka K, Doros G, Wittkopper K, Schulze T, et al. Follistatin-like 1 in chronic systolic heart failure: A marker of left ventricular remodeling. Circ Heart Fail 2011; 4: $621-627$.

80. Hayakawa S, Ohashi K, Shibata R, Takahashi R, Otaka N, Ogawa $\mathrm{H}$, et al. Association of circulating follistatin-like 1 levels with inflammatory and oxidative stress markers in healthy men. PLoS One 2016; 11: e0153619, doi:10.1371/journal.pone.0153619.

81. Gorgens SW, Raschke S, Holven KB, Jensen J, Eckardt K, Eckel J. Regulation of follistatin-like protein 1 expression and secretion in primary human skeletal muscle cells. Arch Physiol Biochem 2013; 119: $75-80$

82. Kuang XL, Zhao XM, Xu HF, Shi YY, Deng JB, Sun GT. Spatiotemporal expression of a novel neuron-derived neurotrophic factor (NDNF) in mouse brains during development. BMC Neurosci 2010; 11: 137 . 